\title{
The Predictors of Parent Reported Behaviors Related to Olfactory Information Processing in Children with ADHD
}

\author{
Ahmad Ghanizadeh \\ Research Center for Psychiatry and \\ Behavioral Sciences, \\ Department of Psychiatry, \\ Shiraz University of Medical Sciences, \\ Hafez Hospital, Shiraz, Iran
}

Objective Attention deficit hyperactivity disorder (ADHD) is a heterogeneous disorder with contradictory findings about smell detection function. It is not clear if the parent perceived behavior related to olfactory function is associated with age, gender, severity of ADHD, and cooccurring symptoms of anxiety and oppositional behavior in children with ADHD.

Methods Participants were a clinical sample of 104 children and adolescents with ADHD using DSM-IV diagnostic criteria by a semi-structured interview. Parent perceived behavior related to olfactory processing function was assessed trough a questionnaire. The parent reported Olfactory Functioning Checklist was used to evaluate "seeking behavior and over-responsiveness to smell (SSBO)" and "smell detection ability (SDA)".

Results ADHD and separation anxiety symptoms count (severity) predicted the SDA scale score. None of the variables of gender, age, ADHD subtypes, co-morbidity with oppositional defiant disorder (ODD), and symptom count of ODD predicted SSBO and SDA scales' scores.

Conclusion Parent reported behavior related to olfactory detection impairment increased with higher ADHD and anxiety severity. It is independent of age and gender.

Psychiatry Investig 2010;7:116-121

Key Words Smell, ADHD, Olfactory Information Processing Checklist, Children, Parent.

Received: January 8, 2010 Revised: March 8, 2010 Accepted: March 15, 2010

Available online: May 4, 2010

\section{Introduction}

Odor identification impairment is reported in many disorders such as schizophrenia, autism, ${ }^{1}$ and frontotemporal dementia. ${ }^{2}$ More impairment of olfactory identification is associated with more likelihood of greater social impairment in children with autism. ${ }^{1}$

Prefrontal cortex is involved in attention deficit hyperactivity disorder (ADHD) ${ }^{3}$ It is also a part of olfactory information processing complex. ${ }^{4}$ Olfactory stimuli activate some regions within the cingulate, orbitofrontal cortex, and ventral insular region, which is continuous with primary olfactory cortex. ${ }^{5}$ Olfactory stimuli evoke the activity of the areas in the brain which are influenced by the subjects' attention. They also evoke activities in the subgenual cingulate and in the central posterior orbitofrontal cortex when the subjects' attention is directed elsewhere but these were not activated when the subjects attended odors. ${ }^{6}$ Olfactory functioning can be used to study the functional integrity of brain regions. Association of ADHD severity and olfactory function in ADHD can raise the possibility that chemosensory dysfunction could serve as a bio-behavioral marker in ADHD. A bio-behavioral marker may represent a clue to genetic underpinnings or change our vision towards psychiatric diagnoses and classification. ${ }^{7}$

ADHD is a cluster of very common symptoms including inattention, impulsivity, and hyperactivity. ${ }^{8} \mathrm{ADHD}$ is usually co-morbid with other psychiatric disorders. More than half of them are co-morbid with oppositional defiant disorder (ODD) and separation anxiety. ${ }^{9}$ ADHD is as- 
sociated with dopamine-induced imbalance of the basal ganglia neurocircuitries. ${ }^{10}$ Dopamine also modulates olfactory processing ${ }^{11}$ while Levodopa does not improve the olfaction in healthy individuals. ${ }^{12}$ Probably, methylphenidate downregulates dopamine turnover. ${ }^{13}$ All of these chemical and neuro-anatomical associations indicate that ADHD and olfactory function may be related to each other.

Another study on adults with ADHD indicated that odor identification ability was not associated with ADHD types or co-morbidity with ODD. ${ }^{14}$ There is scant research with conflicting results on olfactory function in ADHD children. A recently published study reported that the olfactory identification ability of children with ADHD is significantly poorer than that of the healthy controls. They concluded that orbitofrontal regions are compromised in children with ADHD. ${ }^{15}$ The study compared the smell identification ability of children with ADHD and a control group using the Smell Identification Test. The authors mentioned that this study was the first to report significant olfactory function deficit in children with ADHD. However, their sample mainly consisted of the combined type of ADHD (40 children with combined type and 4 children with inattentive type). Moreover, co-morbid psychiatric disorders, intellectual ability, and ADHD severity were not considered. Their result regarding gender was also inconclusive due to the low sample size for girls. Another study reported that stimulants normalized the odor sensitivity threshold in children with ADHD. ${ }^{16}$ Meanwhile, Romanos et al. ${ }^{16}$ found improved odor sensitivity, indicating that olfaction is better in ADHD children compared to controls. So, there is still sufficient reasoning for carrying out studies on olfaction in ADHD.

The processing of pleasant odors in the brain is different from that of unpleasant ones. ${ }^{17}$ Threshold of odor detection increases with age. ${ }^{18}$ Olfactory discrimination is associated with gender and it is much better in boys than girls with ADHD. ${ }^{16}$ However, olfactory identification ability is associated with cognition or intellectual level. ${ }^{14}$ Murphy et al. ${ }^{14}$ indicated that odor identification was no longer significant between ADHD group and control group after controlling for intelligence quotient.

None of the above-motioned studies in ADHD children included severity of ADHD, and the common co-morbid problems of anxiety and oppositional behavior. The studies that included ADHD subtypes included only two types of inattentive and combined type of ADHD, rather than its three types. ${ }^{15,19}$ However, to date, it is not clear whether the severity and type of ADHD, age, gender, the co-morbid problems of oppositional behavior and anxiety are the predictors of behavior related to olfactory function in children and adolescents with ADHD. Both ADHD and smell detection ability (SDA) are associated with prefrontal cortex function. ${ }^{3,14}$ It is hypothesized that more ADHD symptoms counted as the index of ADHD severity is a predicator of higher parent reported olfactory dysfunction in children with ADHD.

\section{Methods}

\section{Participants}

The subjects of this study were a clinical sample of children with ADHD from Shiraz University of Medical Sciences affiliated clinic of child and adolescent psychiatry. They were from a broader ongoing study on sensory processing problems in children with ADHD. ${ }^{20,21}$ Response rate for participation in the study was $95.4 \%$. Five parents refused to take part in the study because it was time-consuming. Eleven parents did not fill the Olfactory Functioning Checklist completely, so they were not included in the analysis. 93 out of 109 children and adolescents were included in the data analysis. Seventy of them were boy and 23 were girl. Their age range was 6 to 14 years with a mean of 8.4 (standard deviation=1.8).

\section{Instruments}

The participants were interviewed using DSM-IV diagnostic criteria ${ }^{8}$ and Farsi version of Schedule for Affective Disorders and Schizophrenia for School-Age Children (K-SADS). ${ }^{22}$ The Farsi version of KSAD is a valid and reliable semi-structured interview that had been used in many other studies. ${ }^{23} \mathrm{Me}-$ dical history was taken and neurological examination was conducted. Children with remarkable problems such as neurological disorder, seizure disorder, pre-maturity, history of recognized meningitis, metabolic disorders, a significant head trauma, and those with of postnatal anoxia were not included. The number of symptoms of criterion A (according to DSMIV) for ADHD, ODD, and SAD was considered as severity of ADHD, ODD, and SAD, respectively.

\section{Olfactory Information Processing Checklist}

There was not found any questionnaire for assessing different aspects of olfactory information processing impairment. So, in the first step of the study, a valid and reliable parent reported Olfactory Functioning Checklist was prepared to assess the behavioral perspective of the two dimensions of the olfactory information processing impairment including "seeking behavior and over-responsiveness to smell (SSBO)" and "SDA", according to the literature. ${ }^{24-27}$

The items of the Checklist that had been used in the abovementioned studies were discussed by three psychiatrists and a psychologist. They confirmed its face and content validity, and the items actually measured what they were supposed to measure.

The Olfactory Functioning Checklist consisted of 14 items rated on a 4-point Likert-type scale ( $0=$ "never or entirely untrue", 1="occasionally", 2="often", to 3="always"). The scale was administered to five parents of children in a child psychiatry clinic to appraise its language and statement comprehensibility. The parents' feedback of the parents was used to modify it. The data included in this manuscript was gathered in compliance with the Helsinki Declaration. Participation in the study 
was voluntary. The parents and children provided oral informed consent and assent, respectively, for participation in the study.

\section{Statistical analysis}

Exploratory factor analysis was conducted using Varimax rotation to examine the construct validity of the scale. Internal consistency was calculated using Cronbach's alpha reliability coefficients. The convergent (range of correlation) and discriminant validities for the items of the scale with the mean scores of SSBO and SDA were examined using Pearson's correlation. Partial correlations were used to examine correlations between ADHD types, ADHD symptoms count, SSBO and SDA after controlling for ODD symptoms count and that of separation anxiety. Two separate linear regression model analyses were conducted to examine the predictors of the two scale scores of SSBO and SDA. Gender, age, ADHD subtypes, and co-morbidity with ODD were considered as independent variables. The number of ADHD symptoms, ODD symptoms, and separation anxiety symptoms were also considered as independent variables. The number of ADHD symptoms was considered as the severity of ADHD. ${ }^{28}$

\section{Results}

\section{Factorial analysis of the Olfactory Information Processing Checklist}

Kaiser-Meyer-Olkin Measure of sampling adequacy was 0.65 and the Bartlett's Test of Sphericity was significant $(\mathrm{p}<$
$0.001)$. The two factors explained $38.3 \%$ of the total variance ( $24.3 \%$ for factor I and $14.0 \%$ for factor II). The eigen values for factors I and II were 3.4 and 1.9, respectively. Two items with item-sum score correlations less than 0.46 were excluded from the related factors. The item of "may drink or eat poisonous substances because she/he cannot detect their bad smell" was deleted to increase the internal reliability. The two factors of SSBO and SDA included 7 and 4 items, respectively (Table 1).

\section{The convergent (range of correlation) and discriminant validities}

Table 2 displays the convergent (range of correlation) and discriminant validities of the checklist. It has enough convergent and discriminant validities.

\section{Internal consistency}

Internal consistency analyses were performed to assess the reliability. Internal consistencies (Cronbach's alpha value) in the whole scale, SSBO scale and SDA scale were 0.73, 0.80, and 0.59 , respectively.

\section{Correlation of variables}

After partialling out for the variables of oppositional defiant behavior and separation anxiety scores, there was no significant correlation between ADHD subtypes, ADHD symptoms count with SSBO scale score (Table 3). Except for ADHD symptoms count, none of them had a significant correlation

Table 1. Factor structure and items loading of the Olfactory Functioning Checklist

\begin{tabular}{lcc}
\hline Items of the scales & \multicolumn{2}{c}{ Factors } \\
\cline { 2 - 3 } Does not like to play in some places because there are smells & SSBO & 0.787 \\
Usually likes sniffing new objects, people, or places & 0.733 & 0.049 \\
Likes some people or some places due to their odor & 0.685 & -0.115 \\
Dislikes or refuses to eat some foods because of their smell & 0.648 & -0.017 \\
Cannot tolerate or is easily disgusted by smell of bathroom or lavatory & 0.601 & 0.044 \\
Dislikes or negatively responds to smells which do not usually bother people & 0.590 & 0.142 \\
To handle the things, he/she sniffs them & 0.065 & 0.810 \\
Cannot detect or recognize bad smells & 0.101 & 0.667 \\
Cannot detect or recognize odors & 0.104 & 0.551 \\
Cannot detect or notice smells that bother others & -0.025 \\
Does not notice or ignore bad smells & 0.512 \\
\hline
\end{tabular}

SSBO: seeking behavior and over-responsiveness to smell, SDA: smell detection ability

Table 2. Range of convergent validity and discriminant validity for the items of the Olfactory Functioning Checklist

\begin{tabular}{lccccc}
\hline Scale & $\begin{array}{c}\text { Number of items } \\
\text { per scale }\end{array}$ & $\begin{array}{c}\text { Convergent validity } \\
\text { (range of correlation) }\end{array}$ & $\begin{array}{c}\text { Discriminant validity } \\
\text { (range of correlation) }\end{array}$ & $\begin{array}{c}\text { Scaling success } \\
\text { (for discriminant validity) }\end{array}$ & $\begin{array}{c}\text { Scaling } \\
\text { success rate }\end{array}$ \\
\hline SSBO & 7 & $0.58-0.76$ & $0.01-0.11$ & $7 / 7$ & $100.0 \%$ \\
SDA & 5 & $0.62-0.72$ & $0.0003-0.11$ & $5 / 5$ & $100.0 \%$ \\
\hline
\end{tabular}

SSBO: seeking behavior and over-responsiveness to smell, SDA: smell detection ability 
Table 3. The correlation of scores of SSBO, SDA, and ADHD after partialling out for the variables of oppositional defiant behavior score and separation anxiety score

\begin{tabular}{llccc}
\hline & & SSBO scale score & ADHD type & ADHD total score \\
\hline SDA scale score & Correlation & 0.036 & 0.131 & 0.429 \\
& Significance (2-tailed) & 0.754 & 0.248 & 0.001 \\
& $\mathrm{df}$ & 78 & 78 & 78 \\
SSBO scale score & Correlation & 1.000 & 0.009 & -0.010 \\
& Significance (2-tailed) & & 0.938 & 78 \\
ADHD type & df & & 78 & 0.927 \\
& Correlation & & 1.000 & 0.164 \\
& Significance (2-tailed) & & 78 \\
\hline
\end{tabular}

SSBO: seeking behavior and over-responsiveness to smell, SDA: smell detection ability, ADHD: attention deficit hyperactivity disorder

Table 4. The mean (standard deviation) score of SSBO, SDA, ADHD symptom count, and anxiety symptoms count by gender

\begin{tabular}{lccrc}
\hline & Total & Boys & Girls & Significance \\
\hline SSBO & $6.9(4.5)$ & $6.9(4.2)$ & $7.0(5.3)$ & $\mathrm{t}=0.1, \mathrm{df}=91, \mathrm{p}=0.8$ \\
SDA & $0.4(0.4)$ & $0.4(0.5)$ & $0.38(0.4)$ & $\mathrm{t}=0.2, \mathrm{df}=91, \mathrm{p}=0.7$ \\
Anxiety score & $2.1(2.0)$ & $2.2(1.9)$ & $1.9(2.2)$ & $\mathrm{t}=0.5, \mathrm{df}=85, \mathrm{p}=0.5$ \\
ADHD score & $32.1(10.1)$ & $32.6(10.2)$ & $30.4(9.7)$ & $\mathrm{t}=0.9, \mathrm{df}=87, \mathrm{p}=0.3$ \\
ODD score & $4.3(2.4)$ & $4.6(2.4)$ & $3.7(2.3)$ & $\mathrm{t}=1.3, \mathrm{df}=91, \mathrm{p}=0.1$ \\
\hline
\end{tabular}

SSBO: seeking behavior and over-responsiveness to smell, SDA: smell detection ability, ADHD: attention deficit hyperactivity disorder, ODD: oppositional defiant disorder

Table 5. Results of general linear model analysis with "smell detection ability" scale score as dependent variable

\begin{tabular}{lcrc}
\hline \multicolumn{1}{c}{ Variable } & df & F & Sig. \\
\hline Age & 1 & 0.036 & 0.851 \\
Separation anxiety symptoms count & 1 & 6.926 & 0.010 \\
Oppositional defiant symptoms count & 1 & 1.976 & 0.164 \\
ADHD symptoms count & 1 & 17.716 & 0.001 \\
Gender & 1 & 1.104 & 0.297 \\
\hline
\end{tabular}

R Squared=0.307 (Adjusted R Squared=0.261). ADHD: attention deficit hyperactivity disorder

with SDA score.

\section{Predictor variables}

The rates of combined, inattentive, and hyperactive/impulsive of $\mathrm{ADHD}$ were $60.2 \%, 10.8 \%$, and $28.4 \%$, respectably. None of the variables of gender (Table 4), age, ADHD subtypes, comorbidity with ODD, scores of ODD, ADHD and separation anxiety predicted SSBO scale score. Table 5 reveals that ADHD severity and separation anxiety symptoms count significantly predicted SDA scale score. However, none of the variables of gender, age, ADHD subtypes, co-morbidity with ODD, and score of ODD predicted SDA scale score.

\section{Discussion}

The current results are in accordance with those of the study on young ADHD adults that indicated odor identification ability was not associated with ADHD types or co-morbidity with ODD ${ }^{14}$ ADHD subtypes are not also distinctive disorders regarding their co-morbidities. ${ }^{29}$ In the current study, none of the ADHD subtypes and ODD co-morbidity was the predictor of the parent perceived behavior related to olfactory processing function in children with ADHD.

Meanwhile, the severity of ADHD is a predictor of SDA. It means that children with more symptoms of ADHD have severer SDA dysfunctions. It might be explained that the association is due to the common cause of children's inattentiveness. In other words, weaker concentration is in association with greater perceptual suppression. ${ }^{30}$ Another explanation is that the children with severer ADHD adapt to smell sooner than those suffering from less severe forms of ADHD and this leads to their lower score of SDA. This smell adaptation might be a type of learning that may help them to accommodate and interact with olfactory environment. ${ }^{30}$

Gender was not associated with both SSBO and SDA. It is not in accordance with the previous study showing that boys more than girls suffered from olfactory impairment. ${ }^{16}$ Of course, that study compared the groups while in this study association of gender with parent perceived behavior related to olfactory function in children with ADHD was studied in a framework of different co-variant variables such as age and cooccurring problems.

Separation anxiety symptoms were also associated with 
SDA score. Previous studies have reported that ADHD patients with high anxiety level misinterpret emotional expressions more than those with lower anxiety level. ${ }^{31}$

All sensory modalities including olfaction require a thalamic relay to consciously analyze a smell. ${ }^{32}$ Olfactory function changes the human olfactory bulb volume over time. ${ }^{33}$ Moreover, attention to odor modulates thalamocortical connectivity in the human brain. ${ }^{32}$ Further studies should survey if interventions on olfactory function in ADHD children such as occupational therapy can impact the ADHD symptoms or find novel approaches to monitoring ADHD.

In the current study, parents reported the smell processing of their children in natural settings. Further studies could be conducted using neurological measures such as electroencephalography and P50 suppression test to evaluate the objective sensory responses. However, smelling of odor in laboratory conditions is a very different experience from when the same odor is smelled naturally because the source of the odor can often be seen in the laboratory condition. Both color and shape information affect olfactory discrimination and there is a multisensory interaction between olfaction and vision. ${ }^{34}$ In other words, visual world can affect perception of the odor. ${ }^{35-37}$ For example, some of the verbal labels could be activated by color because of color-odor association which has already existed. ${ }^{36}$ So, detection of odor is very hard when there is no other cue. Moreover, it is possible that color cues activate olfactory representations. The name of smell, even with lack of smell stimulation, can activate areas of smell-related perceptual processing in the brain. ${ }^{38}$ So, studying behaviors related to smell functioning in natural settings has above-mentioned problems and advantages.

Moreover, neither the current nor the previously mentioned studies considered the mode of olfactory delivery (i.e., orthonasal versus retronasal olfaction). The mode of olfactory delivery may activate different brain areas and networks. ${ }^{5}$ Further studies with larger sample size should include more items for evaluation of smell seeking behavior and the studies can be conducted with separating smell-seeking behaviors and overresponsiveness to smell. Future studies may consider different domains of olfactory function such as olfactory sensitivity, discrimination, and identification. In addition, children with clinically estimated mental retardation were not included in the current study. Further studies may include intellectual functioning, using a formal measurement to assess it.

There is scant research on olfactory function in ADHD children. None of the above-motioned studies in ADHD children included severity of ADHD, and the common problems of anxiety and oppositional behavior. The two studies that included ADHD subtypes included only two types of inattentive and combined types of ADHD, rather than its three types. ${ }^{15,19}$ But the present study included all the three types of ADHD. There was no difference among the three types of ADHD for both of the SSBO and SDA scales scores. These results do not support the idea that the three types of ADHD are distinct regarding neurophysiologic mechanisms of olfactory function in the children with ADHD.

Despite these limitations, the current study has several strengths. It is the first study that investigated parent reported behavior related to olfactory function in the framework of different confounding variables. Secondly, it assessed this subject in a natural setting. Finally, it included the severity of ADHD.

With consideration that there is an olfaction deficit in children with $\mathrm{ADHD}^{15}$ and the association of severity of ADHD and anxiety symptoms with parent reported behavior related to olfactory function, it is noteworthy to survey if modalities restore olfactory function or if intervention on smell function system affects ADHD symptoms.

In summary, this first investigation of association of parent reported behavior related to olfactory function in ADHD children showed that the severity of ADHD is associated with more impaired SDA dysfunction. This association simply cannot be explained as consequences of ADHD or vice verse. It is possible that both of them have unique biological underpinnings or neurologic bases, with implications for diagnostic classification and therapeutic interventions.

\section{- Acknowledgments}

There were no sources or any financial support or relationships or conflict of interest affecting this manuscript to be declared. Part of the data of this study was gathered in accompany with some other published studies. ${ }^{20,21,39}$ The author thanks Drs. Meftagh, Alavi, and Negarestani for their help.

\section{REFERENCES}

1. Bennetto L, Kuschner ES, Hyman SL. Olfaction and taste processing in autism. Biol Psychiatry 2007;62:1015-1021.

2. McLaughlin NC, Westervelt HJ. Odor identification deficits in frontotemporal dementia: a preliminary study. Arch Clin Neuropsychol 2008; 23:119-123.

3. Castellanos FX, Giedd JN, Marsh WL, Hamburger SD, Vaituzis AC, Dickstein DP, et al. Quantitative brain magnetic resonance imaging in attention-deficit hyperactivity disorder. Arch Gen Psychiatry 1996;53: 607-616.

4. Martzke JS, Kopala LC, Good KP. Olfactory dysfunction in neuropsychiatric disorders: review and methodological considerations. Biol Psychiatry 1997;42:721-732.

5. Small DM, Prescott J. Odor/taste integration and the perception of flavor. Exp Brain Res 2005;166:345-357.

6. Sabri M, Radnovich AJ, Li TQ, Kareken DA. Neural correlates of olfactory change detection. Neuroimage 2005;25:969-974.

7. Gottesman II, Gould TD. The endophenotype concept in psychiatry: etymology and strategic intentions. Am J Psychiatry 2003;160:636-645.

8. American Psychiatric Association. Diagnostic and Statistical Manual of Mental Disorders, 4th ed. Washington, DC: American Psychiatric Association; 1994

9. Ghanizadeh A, Mohammadi MR, Moini R. Comorbidity of psychiatric disorders and parental psychiatric disorders in a sample of Iranian children with ADHD. J Atten Disord 2008;12:149-155.

10. Mehler-Wex C, Riederer P, Gerlach M. Dopaminergic dysbalance in distinct basal ganglia neurocircuits: implications for the pathophysi- 
ology of Parkinson's disease, schizophrenia and attention deficit hyperactivity disorder. Neurotox Res 2006;10:167-179.

11. Pavlis M, Feretti C, Levy A, Gupta N, Linster C. 1-DOPA improves odor discrimination learning in rats. Physiol Behav 2006;87:109-113.

12. Rösser N, Berger K, Vomhof P, Knecht S, Breitenstein C, Flöel A. Lack of improvement in odor identification by levodopa in humans. Physiol Behav 2008;93:1024-1029.

13. Ludolph AG, Kassubek J, Schmeck K, Glaser C, Wunderlich A, Buck AK, et al. Dopaminergic dysfunction in attention deficit hyperactivity disorder (ADHD), differences between pharmacologically treated and never treated young adults: a 3,4-dihdroxy-6-[18F]fluorophenyl-1-alanine PET study. Neuroimage 2008;41:718-727.

14. Murphy KR, Barkley RA, Bush T. Executive functioning and olfactory identification in young adults with attention deficit-hyperactivity disorder. Neuropsychology 2001;15:211-220.

15. Karsz FR, Vance A, Anderson VA, Brann PG, Wood SJ, Pantelis C, et al. Olfactory impairments in child attention-deficit/hyperactivity disorder. J Clin Psychiatry 2008;69:1462-1468.

16. Romanos M, Renner TJ, Schecklmann M, Hummel B, Roos M, von Mering C, et al. Improved odor sensitivity in attention-deficit/hyperactivity disorder. Biol Psychiatry 2008;64:938-940.

17. Zald DH, Pardo JV. Emotion, olfaction, and the human amygdala: amygdala activation during aversive olfactory stimulation. Proc Natl Acad Sci U S A 1997;94:4119-4124.

18. Larsson M, Nilsson LG, Olofsson JK, Nordin S. Demographic and cognitive predictors of cued odor identification: evidence from a population-based study. Chem Senses 2004;29:547-554.

19. Gansler DA, Fucetola R, Krengel M, Stetson S, Zimering R, Makary C. Are there cognitive subtypes in adult attention deficit/hyperactivity disorder? J Nerv Ment Dis 1998;186:776-781.

20. Ghanizadeh A. Screening signs of auditory processing problem: does it distinguish attention deficit hyperactivity disorder subtypes in a clinical sample of children? Int J Pediatr Otorhinolaryngol 2009;73:81-87.

21. Ghanizadeh A. Tactile sensory dysfunction in children with ADHD. Behav Neurol 2008;20:107-112.

22. Ghanizadeh A, Mohammadi MR, Yazdanshenas A. Psychometric properties of the Farsi translation of the Kiddie Schedule for Affective Disorders and Schizophrenia-Present and Lifetime Version. BMC Psychiatry 2006;6:10.

23. Ghanizadeh A. ADHD, bruxism and psychiatric disorders: does bruxism increase the chance of a comorbid psychiatric disorder in children with ADHD and their parents? Sleep Breath 2008;12:375-380.

24. Sensory Processing Disorder Checklist. Available at: http://www.sen- sory-processing-disorder.com/sensory-processing-disorder-checklist. html. Accessed January 20, 2008.

25. Larkey S. Practical sensory programmes for students with autism spectrum disorders and other special needs. London and Philadelphia: Jessica Kingsley Publishers; 2007.

26. Emmons PG, Anderson LM. Understanding Sensory Dysfunction. London and Philadelphia: Jessica Kingsley Publishers; 2006.

27. Leekam SR, Nieto C, Libby SJ, Wing L, Gould J. Describing the sensory abnormalities of children and adults with autism. J Autism Dev Disord 2007;37:894-910.

28. Volk HE, Todorov AA, Hay DA, Todd RD. Simple identification of complex ADHD subtypes using current symptom counts. J Am Acad Child Adolesc Psychiatry 2009;48:441-450.

29. Ghanizadeh A. Psychiatric comorbidity differences in clinic-referred children and adolescents with ADHD according to the subtypes and gender. J Child Neurol 2009;24:679-684.

30. Dalton P. Psychophysical and behavioral characteristics of olfactory adaptation. Chem Senses 2000;25:487-492.

31. Williams LM, Hermens DF, Palmer D, Kohn M, Clarke S, Keage H, et al. Misinterpreting emotional expressions in attention-deficit/hyperactivity disorder: evidence for a neural marker and stimulant effects. Biol Psychiatry 2008;63:917-926.

32. Plailly J, Howard JD, Gitelman DR, Gottfried JA. Attention to odor modulates thalamocortical connectivity in the human brain. J Neurosci 2008;28:5257-5267.

33. Haehner A, Rodewald A, Gerber JC, Hummel T. Correlation of olfactory function with changes in the volume of the human olfactory bulb. Arch Otolaryngol Head Neck Surg 2008;134:621-624.

34. Dematté ML, Sanabria D, Spence C. Olfactory discrimination: when vision matters? Chem Senses 2009;34:103-109.

35. Stevenson RJ, Oaten M. The effect of appropriate and inappropriate stimulus color on odor discrimination. Percept Psychophys 2008;70: 640-646.

36. Morrot G, Brochet F, Dubourdieu D. The color of odors. Brain Lang 2001;79:309-320.

37. Gottfried JA, Dolan RJ. The nose smells what the eye sees: crossmodal visual facilitation of human olfactory perception. Neuron 2003;39: 375-386.

38. González J, Barros-Loscertales A, Pulvermüller F, Meseguer V, Sanjuán $\mathrm{A}$, Belloch $\mathrm{V}$, et al. Reading cinnamon activates olfactory brain regions. Neuroimage 2006;32:906-912.

39. Ghanizadeh A. Predictors of postural stability in children with attention deficit hyperactivity disorder. Journal of Attention disorders. In press. 